\title{
Clinical Algorithm for Screening of HIV Among High-risk Children - Need of the Hour!
}

\author{
Ira ShaH* ANd Vishrutha PoOJari \\ Pediatric HIV Clinic, Department of Pediatric Infectious Diseases, B J Wadia Hospital for Children, Mumbai, India. \\ *irashah@pediatriconcall.com
}

$\mathrm{H}$ uman immunodeficiency virus (HIV) transmission in children is most commonly due to vertical transmission [1]. Under the Prevention of parent to child transmission (PPTCT) program, all pregnant women are screened for HIV, which enables early diagnosis of HIV-exposed infants as they are at high risk for malnutrition, growth failure, developmental delay and repeated infections with common as well as uncommon organisms [1]. Without treatment, about one third of infants living with HIV will die in their first year and $50 \%$ by the second year of life [1]. The National HIV program provides access to early diagnosis for HIV testing of infants and children younger than 18 months who are HIV-exposed, and ensures that they receive the required essential package of care as part of the country's commitment on achieving 90-90-90 target by 2030, which aims at ending the acquired immunodeficiency syndrome (AIDS) epidemic [1]. In an attempt to achieve the target, screening of sick infants/ children with unknown HIV exposure is important.

Perinatally-infected adolescents are more likely to suffer from chronic diseases, neurodevelopmental delay, growth and pubertal delays, unlike adolescents who acquire HIV behaviorally [1]. In 2014, it was estimated that $15 \%$ of all persons living with HIV in United States had undiagnosed HIV infection [2]. As per the US Preventive Services Task Force Recommendation (USPSTF) recommendation, persons aged 15-65 years should be screened for HIV at least once, and younger adolescents and older adults at increased risk should also be screened [3]. As per World Health Organization (WHO) 2010 guideline, it is strongly recommended to use the clinical algorithm and serologic test in the absence of virologic testing in sick infants for presumptive clinical diagnosis of HIV infection [4]. The adverse social and economic factors like poverty, broken families, parental sickness/drug abuse, and stigmatization by the society are the factors hindering access to medical care [1]. Integrated Management of Neonatal and Childhood Illness (IMNCI), which is adapted from the global version of Integrated Management of Childhood Illness (IMCI), is a strategy to address high infant mortality and to meet sustainable developmental goals with target of reducing under five mortality to 25 per 1000 live birth $[5,6]$. There are mainly seven clinical features included in the IMCI/HIV algorithm for the clinical diagnosis of HIV infection in children - pneumonia, persistent diarrhea, ear discharge (acute or chronic), very low weight for age, oral thrush, parotid enlargement, and generalized persistent lymphadenopathy [7].

In an African study [8], the performance of the IMCI HIV algorithm in a cohort of 444 HIV-exposed Kenyan infants was studied. The overall sensitivity, specificity, positive predictive value (PPV) and negative predictive value (NPV) were $58 \%, 87 \%, 52 \%$ and $90 \%$, respectively. It was noted that sensitivity was lowest at 1 month of age, when majority of HIV infections already had occurred and initiation of treatment is most important. The use of IMCI was estimated to delay diagnosis in HIV-infected infants by a median of 5.9 months. Oral thrush (67\%), lymphadenopathy $(55 \%)$ and pneumonia $(55 \%)$ were the most commonly identifiedfeatures in HIV-1 infected infants. However, IMCI still is useful in identifying older children with undiagnosed HIV-1 infection, acquiring infection through late breastmilk transmission [8]. Sensitivity and specificity estimates of HIVclinical algorithms over various studies have ranged from $9-89 \%$ and $42-99 \%$, respectively [8].

Integrated Management of Adolescence and Adult Illness (IMAI) is a facility level health care service, which presents a syndromic case management protocol to diagnose and manage common adult illnesses [9]. The sensitivity and specificity of IMAI acute care algorithm in a HIV positive Ethiopian cohort was above $85 \%$ and above $92 \%$, respectively [10].

In this issue of Indian Pediatrics, Sinha, et al. [11] present a cross-sectional study on the utility of Indian Council of Medical research (ICMR) modified integrated 
algorithm as a screening tool in sick children for pediatric HIV case detection in health care facilities. The WHO generic IMCI-HIV screening algorithm for children up to 5 years of age, modification from Integrated Management of Adolescence and Adult Illness for children 5-14 years of age and 'other clues' for all children which includes risk factors and certain clinical conditions of WHO staging of HIV infection were used as screening tools. The HIV prevalence estimated in this study was $19.1 \%$ ( $5 \%$ in $<5$ years and $28 \%$ in $5-14$ years), which is high, and is attributed to screening of sick children. The important predictors of HIV infection noted in this study were parents with HIV, unexplained fever ( $>1$ month) and orphaned child. The strength of this study is the use of standard algorithms from IMCI HIV algorithm and IMAI which were modified as screening tools [11]. However, this screening algorithm could not be validated.

This study [11] is a multicentric study, but the population was limited to one state. Authors highlight the need for routine surveillance of HIV infection amongst children aged 5-14 years considering the high proportion of this population [11]. They concluded that one should have a high index of suspicion to consider the clinical diagnosis of HIV infection, when an infant/child with unknown HIV status butwithrisk factors like orphaned child, child having a single parent, child with high risk behavior, presents with symptoms and/or signs as per the clinical algorithm of WHO-IMCI. However, since this study included children predominantly above 1 year of age, the validity of algorithm in infants, especially HIVexposed infants, needs to be evaluated.

The need of the hour is to have an estimation of HIV disease burden in symptomatic infants/children with risk factors at community level. It is important to understand that the modified integrated algorithm is a screening tool and not a diagnostic test. It is important to sensitize the healthcare workers regarding the use of this algorithm with appropriate training. The utility of this modified integrated algorithm needs to be further tested in different field studies in different states of India to confirm that these results are replicated.

Competing interest: None stated; Funding: Nil.

\section{REFERENCES}

1. National AIDS Control Organization (NACO). National Technical Guidelines on Antiretroviral Treatment,
October, 2018. Available at: https://lms.naco.gov.in/ frontend/content/NACO\%20-\%20National\% 20Technica l\%20Guidelines\%20on\%20ART_October\%202018 $\% 20(1)$.pdf. Accessed on 25 May, 2020.

2. Centers for Disease Control and Prevention (CDC). Monitoring selected national HIV prevention and care objectives by using HIV surveillance data- United States and 6 dependent areas, 2015. HIV Surveillances Supplemental Report 2017. Available at: http:// www.cdc.gov/hiv/library/reports/hiv-surveillance.html. Accessed on 25 May, 2020

3. US Preventive Services Task Force, Owens DK, Davidson KW, Krist AH, Barry MJ, Cabana M, et al. Screening for HIV Infection: US Preventive Services Task Force Recommendation Statement. JAMA. 2019;321:2326-36.

4. World Health Organization. WHO Recommendations on the Diagnosis of HIV Infection in Infants and Children, 2010. Available from: https://apps.who.int/iris/bitstream/ handle/10665/44275/9789241599085_eng.pdf; jsessionid $=5$ C535A56F483F12 DA250B53F3934482F? sequence $=1$. Accessed on 25 May, 2020

5. Mohan P, Kishore B, Singh S, Bahl R, Puri A, Kumar R. Assessment of implementation of integrated management of neonatal and childhood illness in India. J Health PopulNutr. 2011;29:629-38.

6. Aneja S. Integrated management of newborn and childhood illness (IMNCI) strategy and its implementation in real life situation. Indian J Pediatr. 2019;86:622 7.

7. World Health Organization. Integrated Management of Childhood Illness Complementary Course on HIV/AIDS 2006. Available at: http://whqlibdoc.who.int/publications/ 2008/9789241597388_eng.pdf. Accessed on 25 May, 2020.

8. Diener LC, Slyker JA, Gichuhi C, Tapia KA, Richardson $\mathrm{BA}$, Wamalwa $\mathrm{D}$,et al. Performance of the integrated management of childhood illness algorithm for diagnosis of HIV-1 infection among African infants. AIDS. 2012;26:1935 41.

9. World Health Organization. Integrated Management of Adolescent and Adult Illness: Interim Guidelines for FirstLevel Facility Health Workers at Health Centre and District Outpatient Clinic: Acute Care, 2009. Available at: http:// www.who.int/hiv/pub/imai/primary_acute/en/index.html. Accessed on 25 May, 2020

10. Woldie M, Enquselassie F. Assessment of the validity of the guideline for integrated management of adult and adolescent illnesses on HIV patients in Addis Ababa, Ethiopia. Ethiop Med J. 2009;47:195 203.

11. Sinha A, Washington R, Sethumadhavan R, Perumal V, Potty RS, Isac S. Modified integrated algorithm for detection of HIV among sick children aged 0-14 year seeking care at healthcare facilities in India. Indian Pediatr. 2020;57:631-5. 